\title{
Subcortical mapping of calculation processing in the right parietal lobe
}

\author{
Alessandro Della Puppa, MD, ${ }^{1}$ Serena De Pellegrin, ST, ${ }^{2}$ Anna Lazzarini, ST, ${ }^{2}$ Giorgio Gioffrè, MD, ${ }^{1}$ \\ Oriela Rustemi, MD, ${ }^{1}$ Annachiara Cagnin, MD, ${ }^{2}$ Renato Scienza, MD, ${ }^{1}$ and Carlo Semenza, MD ${ }^{2}$
}

Departments of ${ }^{1}$ Neurosurgery and ${ }^{2}$ Neurosciences: Sciences NPSRR, Padua University Hospital, Padua, Italy

\begin{abstract}
Preservation of calculation processing in brain surgery is crucial for patients' quality of life. Over the last decade, surgical electrostimulation was used to identify and preserve the cortical areas involved in such processing. Conversely, subcortical connectivity among different areas implicated in this function remains unclear, and the role of surgery in this domain has not been explored so far. The authors present the first 2 cases in which the subcortical functional sites involved in calculation were identified during right parietal lobe surgery. Two patients affected by a glioma located in the right parietal lobe underwent surgery with the aid of MRI neuronavigation. No calculation deficits were detected during preoperative assessment. Cortical and subcortical mapping were performed using a bipolar stimulator. The current intensity was determined by progressively increasing the amplitude by $0.5-\mathrm{mA}$ increments (from a baseline of $1 \mathrm{~mA}$ ) until a sensorimotor response was elicited. Then, addition and multiplication calculation tasks were administered. Corticectomy was performed according to both the MRI neuronavigation data and the functional findings obtained through cortical mapping. Direct subcortical electrostimulation was repeatedly performed during tumor resection. Subcortical functional sites for multiplication and addition were detected in both patients. Electrostimulation interfered with calculation processing during cortical mapping as well. Functional sites were spared during tumor removal. The postoperative course was uneventful, and calculation processing was preserved. Postoperative MRI showed complete resection of the tumor. The present preliminary study shows for the first time how functional mapping can be a promising method to intraoperatively identify the subcortical functional sites involved in calculation processing. This report therefore supports direct electrical stimulation as a promising tool to improve the current knowledge on calculation processing connectivity.
\end{abstract}

http://thejns.org/doi/abs/10.3171/2014.10.JNS14261

KEY WORDS subcortical mapping; awake surgery; electrostimulation; addition; multiplication; calculation processing connectivity; functional neurosurgery

$\mathrm{P}$ RESERVATION of calculation processing during brain surgery is crucial for patients' quality of life. Over the last decade, surgical electrostimulation proved effective in identifying and preserving the areas involved in calculation processing in the left hemisphere. ${ }^{13}$ This method has been recently validated in the right cortex as well. ${ }^{4}$ Subcortical connectivity among different areas involved in calculation processing remains unclear, and the role of surgery in this domain has not yet been explored. We present the first report concerning the subcortical mapping of calculation processing during resection of brain tumors in the right parietal lobe.

\section{Methods}

\section{Neuropsychological Assessment and Surgical Strategy}

Because of the location of the tumor in the right parietal lobe, awake intraoperative functional mapping was performed before and during resection, to reduce the risk of unfavorable outcomes on calculation. Patients gave their written informed consent. A full assessment of calculation skills was performed before surgery according to the methods of Delazer et al., ${ }^{3}$ assessing different counting abilities, number comprehension, numerical transcoding, calculation, arithmetic reasoning, and conceptual knowl-

ABBREVIATIONS IPL = inferior parietal lobule; $\mathrm{SPL}=$ superior parietal lobule.

SUBMITTED February 4, 2014. ACCEPTED October 16, 2014.

INCLUDE WHEN CITING Published online November 21, 2014; DOI: 10.3171/2014.10.JNS14261.

DISCLOSURE The authors report no conflict of interest concerning the materials or methods used in this study or the findings specified in this paper. 
edge. Calculation abilities included simple fact retrieval, rule-based processing, and mental and written calculation in all 4 operations. Over the 2 preoperative days, the patients were informed in detail about the intraoperative monitoring procedures, were introduced to the stimulation devices, and were trained to perform the naming and calculating tasks. Furthermore, full pre- and postoperative neuropsychological assessments were performed (Table 1). Intraoperatively, cortical and subcortical mapping was performed using a bipolar stimulator. A functional cortical map was obtained using the method described by Duffau et al. ${ }^{5}$ Calculation tasks were administered as previously reported. ${ }^{4}$ A bipolar probe with 5-mm-spaced tips delivering a biphasic current was applied to the cortex for a period of 4 seconds (with a pulse frequency of $60 \mathrm{~Hz}$ and a single pulse phase duration of $0.3 \mathrm{msec}$ ). The intensity of stimulation was determined through progressive increases by $0.5 \mathrm{~mA}$ (from a baseline of $1 \mathrm{~mA}$ ) until a sensorimotor response was obtained. Finally, calculation tasks were administered. Each cortical site $(5 \times 5 \mathrm{~mm})$ of the entire cortex exposed by the bone flap was tested 3 times. Corticectomy was then performed according to both the MRI neuronavigation data and the functional findings acquired during cortical mapping. Direct subcortical electrostimulation was repeatedly performed during tumor resection at the same current intensity used at the cortical level. Sites were marked with tags as functional for calculation when an error was detected at 3 repeated stimulations. The resection was stopped according to the functional corticalsubcortical boundaries provided by electrostimulation.

\section{Calculation Tasks}

All numerical stimuli were presented visually as Arabic numerals on the computer screen, set by the patient at a distance of about $20 \mathrm{~cm}$ (Fig. 1). Tasks were presented on a white background and gray frame to reduce visual fatigue. Due to time constraints, only addition and multiplication were studied. Two different types of calculation tasks were administered to the patient: 1-digit addition with 1 operand (for example, $4+7,8+6$, and $5+7$ ) and 1-digit multiplication with 1 operand (for example, $8 \times 4$, $5 \times 6$, and $9 \times 7$ ).

Each math problem had to be solved within the 4-second time of the cortical stimulation and was presented in the middle of the screen without the equals sign; the answer was oral. The patient was not aware of when the electrical stimulation was performed. The task administration procedure was as follows: a block of 14 addition problems was presented to the patient, in random order, alternating the use of electrostimulation and repeated 3 times. On each stimulation site, the patient performed 3 addition problems, for a total of 21 trials with and 21 trials without stimulation. A block of 15 multiplication problems was then administered (and repeated 3 times) in the same fashion, for a total of 22 tests with and 23 without stimulation. A speech therapist monitored the type of errors.

\section{Results}

\section{Patients}

The clinical and radiological data are summarized in Table 2. Both patients were right-handed. ${ }^{9}$ In both cases
TABLE 1. Pre- and postoperative neuropsychological test protocol

\begin{tabular}{|c|c|}
\hline Test & Cognitive Ability \\
\hline \multicolumn{2}{|l|}{ Language } \\
\hline \multicolumn{2}{|l|}{ Subtest of AAT } \\
\hline Token test & $\begin{array}{l}\text { Language comprehension, severity of } \\
\text { language disorder }\end{array}$ \\
\hline Repetition & $\begin{array}{l}\text { Repetition phonemes, words, \& sen- } \\
\text { tences }\end{array}$ \\
\hline Written language & Reading, writing words \& sentences \\
\hline Picture naming & Naming (word finding) \\
\hline DO-80 & Naming (word finding) \\
\hline \multicolumn{2}{|c|}{ Other cognitive function } \\
\hline ENB & $\begin{array}{l}\text { Attention, visuospatial function, execu- } \\
\text { tive function, memory, praxis }\end{array}$ \\
\hline Calculation skills & $\begin{array}{l}\text { Counting skills, number comprehension, } \\
\text { numerical transcoding, calculation }\end{array}$ \\
\hline \multicolumn{2}{|c|}{ Quality of life \& mood state* } \\
\hline EORTC QLQ-C30 & Quality of life \\
\hline HADS & Anxiety \& depression scale \\
\hline
\end{tabular}

AAT $=$ Aachener Aphasia Test; DO-80 $=$ Test de Dénomination Orale D'images; ENB = Esame Neuropsicologico Breve; EORTC QLQ-C30 = European Organisation for Research and Treatment of Cancer Quality of Life Questionnaire-C30; HADS = Hospital Anxiety and Depression Scale.

* Only assessed preoperatively.

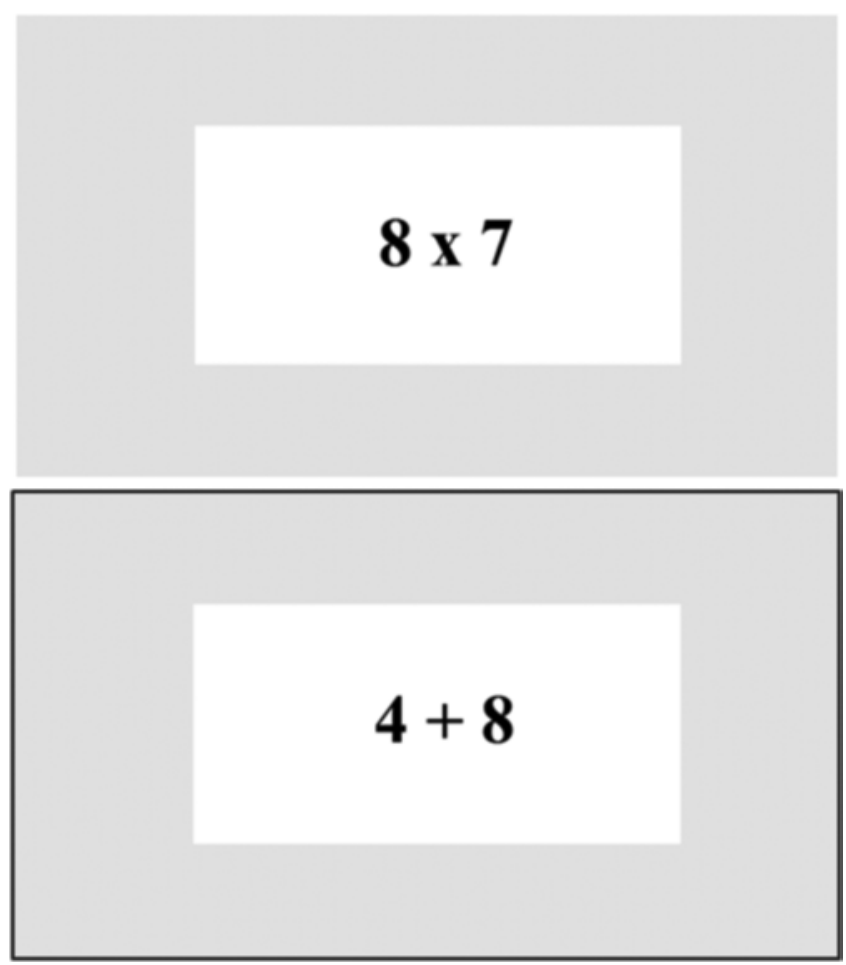

FIG. 1. Example of calculation task administered during intraoperative mapping. 
TABLE 2. Clinical findings in the patients

\begin{tabular}{ccccccc}
\hline $\begin{array}{c}\text { Case } \\
\text { No. }\end{array}$ & $\begin{array}{c}\text { Age }(\mathrm{yrs}), \\
\text { Sex }\end{array}$ & Clinical Presentation & $\begin{array}{c}\text { Preop Calculation } \\
\text { Deficit }\end{array}$ & Tumor Location & Pathology & Postop Calculation Deficit \\
\hline 1 & $57, \mathrm{M}$ & Seizure & No & Right parietal & High-grade glioma & No \\
\hline 2 & $67, \mathrm{~F}$ & Left-hand paresis & No & Right parietal & High-grade glioma & No \\
\hline
\end{tabular}

preoperative MRI showed a high-grade glioma in the white matter of the right parietal lobe (Figs. 2A and 3A).

\section{Preoperative and Postoperative Assessment}

Preoperative neuropsychological examination findings were normal in both cases (Table 2). Neither language impairment nor emotional problems were detected before the operation. ${ }^{7}$ The assessment of calculation skills ${ }^{3}$ showed a normal performance. Visuospatial function, executive function, memory, and praxis as well as general cognitive functions were intact as assessed by specific tests. ${ }^{8}$ Both patients showed a normal performance at postoperative neuropsychological assessment.

\section{Intraoperative Functional Data}

Intraoperative data are summarized in Table 3.

\section{Case 1}

A sensorimotor response was elicited at $2.0 \mathrm{~mA}$. The functional stimulation mapping resulted in the identification of the following cortical and subcortical sites in the parietal lobe: the primary sensorimotor area (Tag 1); 1 cortical site was involved in addition, located within the anterior part of the inferior parietal lobule (IPL) cortex $($ Tag +$)$; 1 cortical site was involved in multiplication, located within the anterior part of the IPL cortex (Tag x); 2 subcortical sites were involved in multiplication, all located at the anterior part of the resection cavity within the
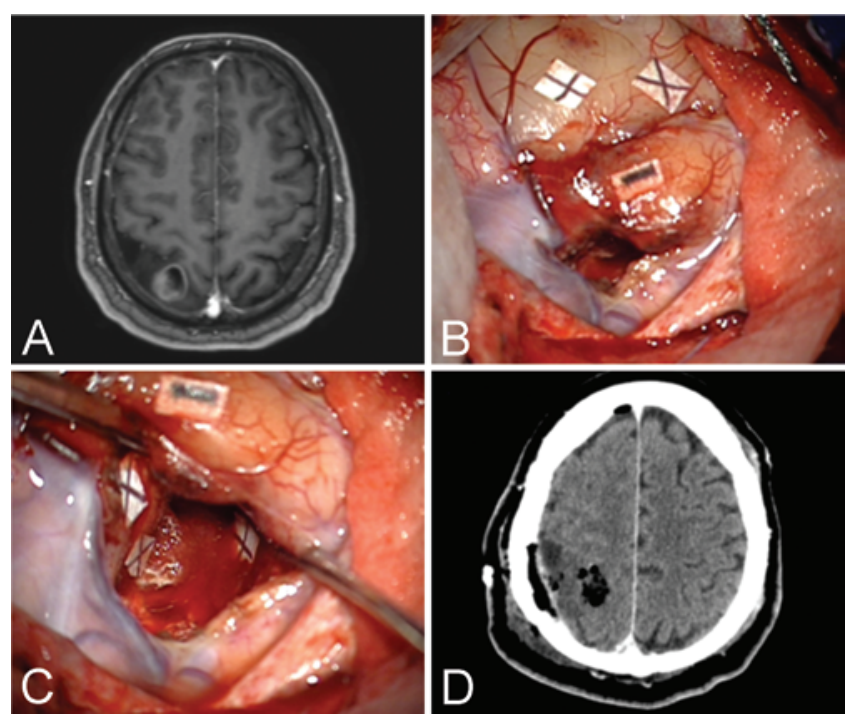

FIG. 2. Case 1. A: Preoperative T1-weighted Gd-enhanced MR image showing a tumor in the right parietal lobe. B and C: Intraoperative views showing cortical $(\mathrm{B})$ and subcortical $(\mathrm{C})$ mapping data. D: Postoperative CT scan (MRI was not performed because of the patient's claustrophobia). Figure is available in color online only. superior parietal lobule (SPL) white matter (Tags x); and 1 subcortical site was involved in addition, located at the posterior part of the resection cavity within the SPL white matter (Tag +) (Fig. 2). No site was positive for both addition and multiplication.

\section{Case 2}

A sensorimotor response was elicited at $2.5 \mathrm{~mA}$. The functional stimulation mapping resulted in the identification of the following cortical and subcortical sites in the parietal lobe: the primary sensorimotor areas (Tags 1-3); 1 cortical site was involved in multiplication, located within the anterior part of the SPL cortex (Tag x); 1 cortical site was involved in addition, located within the anterior part of the SPL cortex (not shown); 2 subcortical sites were involved in multiplication and were located in the posterior part of the resection cavity within the SPL white matter (Tags $\mathrm{x}$ ); and 1 subcortical site was involved in addition, located in the posterior part of the resection cavity within the SPL white matter (Tag +) (Fig. 3). No site was positive for both addition and multiplication.

\section{Postoperative Course}

The postoperative course was uneventful, and calculation processing was preserved in both patients. The patients underwent postoperative CT scanning (Fig. 2D) and MRI (Fig. 3D) (MRI was not performed in Case 1 because of the patient's claustrophobia), showing the complete removal of the tumor.
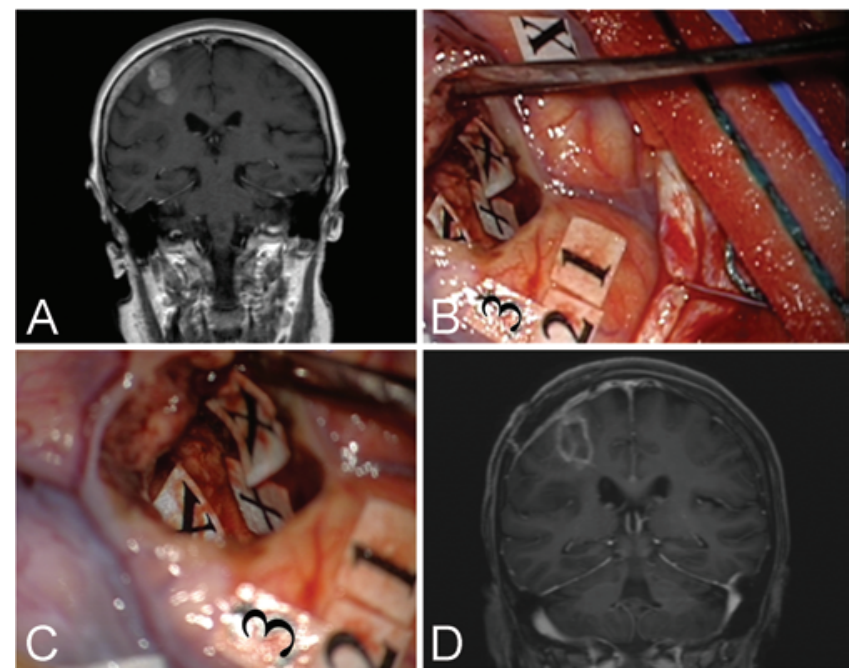

FIG. 3. Case 2. A: Preoperative T1-weighted Gd-enhanced MR image showing a tumor in the right parietal lobe. $\mathbf{B}$ and $\mathrm{C}$ : Intraoperative views showing cortical $(B)$ and subcortical $(C)$ mapping data. D: Postoperative T1-weighted Gd-enhanced MR image. Figure is available in color online only. 
TABLE 3. Intraoperative mapping data

\begin{tabular}{|c|c|c|c|c|c|c|}
\hline \multirow[b]{3}{*}{ Case No. } & \multicolumn{3}{|c|}{ Cortical Sites } & \multicolumn{3}{|c|}{ Subcortical Sites } \\
\hline & \multirow[b]{2}{*}{ SM } & \multicolumn{2}{|c|}{ Calculation } & \multirow[b]{2}{*}{ SM } & \multicolumn{2}{|c|}{ Calculation } \\
\hline & & Addition & Multiplication & & Addition & Multiplication \\
\hline 1 & 1 & 1 (IPL) & 1 (IPL) & 0 & 1 & 2 \\
\hline 2 & 3 & 1 (SPL) & 1 (SPL) & 0 & 1 & 2 \\
\hline Total & 4 & 2 & 2 & 0 & 2 & 4 \\
\hline
\end{tabular}

$\mathrm{SM}=$ sensorimotor.

\section{Discussion}

Awake subcortical direct stimulation has become the gold-standard technique to assist surgery in eloquent areas when cognitive functions might be involved. Over the last decade different tasks have been proven effective in testing different cognitive functions during awake surgery. ${ }^{6}$ Calculation processing may be monitored during surgery in different areas, such as left frontal, temporal, and parietal cortices. ${ }^{5,11,13}$ As far as the right hemisphere is concerned, we recently confirmed previous neuroimaging $^{1,2,10,12}$ and transcranial magnetic stimulation ${ }^{14}$ data, suggesting a role of the right cortex in calculation processing. ${ }^{4}$ Conversely, no surgical data have been reported in the literature on subcortical mapping of right parietal white matter so far. In our 2-case series, we interfered with calculation processing by performing electrostimulation in the white matter of the right parietal lobe during addition and multiplication tasks. To the best of our knowledge, such interference has never been reported before. In both patients we detected errors in addition and multiplication processing at both cortical and subcortical mapping. In neither case was a subcortical deficit detected in the absence of a cortical stimulation-related deficit. An anatomical relationship between cortical- and subcortical deficit-related sites was observed in the patient in Case 2, in whom we found a close connection between the cortical and subcortical areas involved in multiplication (Fig. 3B and C), all within the SPL. Finally, this small cohort of patients cannot provide sufficient data on the clinical efficacy of the present approach. However, the present study shows, for the first time, how subcortical mapping can be a promising method to identify intraoperatively the subcortical functional sites involved in calculation processing. This report therefore further supports direct electrical stimulation as a promising tool to improve the current knowledge on calculation processing connectivity.

\section{References}

1. Arsalidou M, Taylor MJ: Is $2+2=4$ ? Meta-analyses of brain areas needed for numbers and calculations. Neuroimage 54:2382-2393, 2011

2. Dehaene S, Molko N, Cohen L, Wilson AJ: Arithmetic and the brain. Curr Opin Neurobiol 14:218-224, 2004
3. Delazer M, Girelli L, Granà A, Domahs F: Number processing and calculation-normative data from healthy adults. Clin Neuropsychol 17:331-350, 2003

4. Della Puppa A, De Pellegrin S, d'Avella E, Gioffrè G, Munari M, Saladini M, et al: Right parietal cortex and calculation processing: intraoperative functional mapping of multiplication and addition in patients affected by a brain tumor. $\mathbf{J}$ Neurosurg 119:1107-1111, 2013

5. Duffau H, Denvil D, Lopes M, Gasparini F, Cohen L, Capelle $\mathrm{L}$, et al: Intraoperative mapping of the cortical areas involved in multiplication and subtraction: an electrostimulation study in a patient with a left parietal glioma. J Neurol Neurosurg Psychiatry 73:733-738, 2002

6. Fernández Coello A, Moritz-Gasser S, Martino J, Martinoni M, Matsuda R, Duffau H: Selection of intraoperative tasks for awake mapping based on relationships between tumor location and functional networks. J Neurosurg 119:13801394,2013

7. Luzzatti C, De Blaser R: Morphological processing in Italian agrammatic speakers: eight experiments in lexical morphology. Brain Lang 54:26-74, 1996

8. Mondini S, Mapelli D, Vestri A, Bisiacchi PS: Esame Neuropsicologico Breve. Milan: Raffaello Cortina Editore, 2003

9. Oldfield RC: The assessment and analysis of handedness: the Edinburgh inventory. Neuropsychologia 9:97-113, 1971

10. Price GR, Mazzocco MM, Ansari D: Why mental arithmetic counts: brain activation during single digit arithmetic predicts high school math scores. J Neurosci 33:156-163, 2013

11. Pu S, Li YN, Wu CX, Wang YZ, Zhou XL, Jiang T: Cortical areas involved in numerical processing: an intraoperative electrostimulation study. Stereotact Funct Neurosurg 89:42-47, 2011

12. Rosenberg-Lee M, Chang TT, Young CB, Wu S, Menon V: Functional dissociations between four basic arithmetic operations in the human posterior parietal cortex: a cytoarchitectonic mapping study. Neuropsychologia 49:2592-2608, 2011

13. Roux FE, Boukhatem L, Draper L, Sacko O, Démonet JF: Cortical calculation localization using electrostimulation. J Neurosurg 110:1291-1299, 2009

14. Salillas E, Semenza C, Basso D, Vecchi T, Siegal M: Single pulse TMS induced disruption to right and left parietal cortex on addition and multiplication. Neuroimage 59:31593165,2012

\section{Author Contributions}

Conception and design: Della Puppa, De Pellegrin, Semenza. Acquisition of data: Della Puppa, De Pellegrin, Lazzarini. Analysis and interpretation of data: Della Puppa, De Pellegrin, Lazzarini, Cagnin. Drafting the article: Della Puppa. Critically revising the article: Della Puppa, Gioffrè, Rustemi, Cagnin, Scienza, Semenza. Reviewed submitted version of manuscript: Della Puppa, Gioffrè, Rustemi, Scienza. Approved the final version of the manuscript on behalf of all authors: Della Puppa.

\section{Correspondence}

Alessandro Della Puppa, Department of Neurosurgery, Padua University Hospital, Azienda Ospedaliera di Padova, via Giustiniani 2, 35128 Padova, Italy. email: alessandro. dellapuppa@sanita.padova.it. 